\title{
Biochemical Bone Turnover Markers and Osteoporosis in Older Men: Where Are We?
}

\author{
Pawel Szulc \\ INSERM UMR 1033, Université de Lyon, Hôpital Edouard Herriot, Pavillon F, Place d'Arsonval, 69437 Lyon, France \\ Correspondence should be addressed to Pawel Szulc, pawel.szulc@inserm.fr
}

Received 29 May 2011; Revised 8 November 2011; Accepted 12 November 2011

Academic Editor: S. Minisola

Copyright () 2011 Pawel Szulc. This is an open access article distributed under the Creative Commons Attribution License, which permits unrestricted use, distribution, and reproduction in any medium, provided the original work is properly cited.

In men aged less than 60, the association of serum and urinary levels of biochemical bone turnover markers (BTMs) and bone mineral density (BMD) is weak or not significant. After this age, higher BTM levels are correlated weakly, but significantly, with lower BMD and faster bone loss. Limited data from the cohort studies suggest that BTM measurement does not improve the prediction of fragility fractures in older men in comparison with age, BMD, history of falls and fragility fractures. Testosterone replacement therapy (TRT) decreases bone resorption. During TRT, bone formation markers slightly increase (direct effect on osteoblasts), then decrease (slowdown of bone turnover). Bisphosphonates (alendronate, risedronate, ibandronate, zoledronate) induce a rapid decrease in bone resorption followed by a milder decrease in bone formation. In men receiving antiresorptive therapy for prostate cancer, zoledronate, denosumab and toremifene decrease significantly levels of bone resorption and bone formation markers. Teriparatide induced a rapid increase in serum concentrations of bone formation markers followed by an increase in bone resorption. We need more studies on the utility of BTM measurement for the improvement of the persistence and adherence to the anti-osteoporotic treatment in men.

\section{Introduction}

Biochemical markers of bone turnover (BTMs) have been investigated in several male cohorts for the last 15 years. These studies provided data on age-related changes in bone turnover rate in men and on potential determinants of bone turnover rate in men, such as hormones, lifestyle factors, diseases, or medications. However, data on practical use of BTM in the clinical management of osteoporosis in men are limited and rather disappointing.

There are two groups of biochemical bone turnover markers (BTMs), markers of bone formation and markers of bone resorption (Table 1). OC, PICP, and PINP are released during the synthesis of OC and type I collagen which are constituents of bone matrix. CTX-I, NTX-I, PDP, and PYD are products of catabolism of type I collagen [1]. Bone $\mathrm{AP}$ and TRACP5b are enzymes reflecting the metabolic activity, respectively, of osteoblasts and osteoclasts. Recently, it has been proposed that PINP and serum CTX-I become the referent markers of bone formation and resorption, respectively [2].

\section{Association of BTM Levels with Bone Mineral Density and Bone Loss in Men}

The association between BTM levels and areal bone mineral density ( $\mathrm{aBMD}$ ) measured by dual energy $\mathrm{X}$-ray absorptiometry (DXA) was assessed in cross-sectional studies. Before the age of 60 , the association was nonsignificant [3]. It may reflect two processes. In young adult men, the consolidation, that is, formation of peak aBMD after the growth arrest, is associated with a slowdown of bone turnover. However, one BTM level cannot reflect different trends which vary according to the skeletal site. In middle-aged men, apparent stability of aBMD is a trade-off between periosteal apposition and endosteal bone loss. As these processes are slow, aBMD may depend more on the peak aBMD acquired previously than on the current bone turnover rate.

After the age of 60, BTM levels were correlated moderately negatively with aBMD $[3,4]$. The difference in average aBMD between the lowest and the highest BTM quartiles varied from 3 to $12 \%$. Prospective data on the association between BTM levels and bone loss in men are scanty and, 
TABLE 1: Biochemical bone turnover markers.

Bone formation
Osteocalcin $(O C)$
Bone alkaline phosphatase (bone ALP)
N-terminal propeptides of type I procollagen (PINP)
C-terminal propeptides of type I procollagen (PICP)
Bone resorption
C-terminal cross-linking telopeptides of type I collagen
(CTX-I),
N-terminal cross-linking telopeptides of type I collagen
(NTX-I)
C-terminal cross-linking telopeptide of type I collagen
generated by matrix metalloproteinases (CTX-MMP, ICTP)
Helical peptide $620-633$ of the al chain
Deoxypyridinoline $(D P D)$
Pyridinoline $(P Y D)$
Isoform $5 \mathrm{~b}$ of tartrate resistant acid phosphatase $(T R A C P 5 b)$

most often, negative. Higher BTM levels were associated with faster bone loss at some skeletal sites (e.g., hip, distal forearm), but not all (e.g., spine) [5-7].

Several factors can contribute to these results. Bone loss is slower in men than $\mathrm{n}$ women, especially before the age of 70. Thus, a long-term follow-up is necessary to obtain an accurate estimate of bone loss. The rate of bone loss may vary according to the skeletal site. In particular, lumbar spine is not a reliable skeletal site for estimation of bone loss in men because of frequent osteoarthritis. The assessment of bone loss (which is in fact the endosteal bone loss) is biased by the concomitant periosteal apposition. This process is present in both older men and women; however, it may have a greater impact in men who have lower endosteal bone loss and greater periosteal apposition than in women. Finally, bone turnover is influenced by many factors and a single measurement of BTM may not correspond to the average bone turnover rate over a long period of time.

\section{Association between BTM Levels and the Risk of Fracture in Men}

Several prospective cohort and case-control studies showed that elevated BTM levels predict fractures in postmenopausal women after adjustment for age, aBMD, and history of fracture. This association was found mainly in follow-ups lasting $<5$ years, mainly for bone resorption markers, and mainly for major osteoporotic fracture (mainly hip fracture) $[1,7]$. Fewer studies concerned the prediction of fractures by BTM in men. The cohort studies (MrOS, MINOS) showed that, in older men, higher BTM levels did not predict fractures in models adjusted for age and aBMD $[5,6]$. Two studies suggested that BTMs predict fractures in men. However, the association between low gammacarboxylation of osteocalcin (OC) and fracture risk was not adjusted for aBMD [8]. In the Dubbo cohort, only C-terminal crosslinking telopeptide of type I collagen generated by metalloproteinase (ICTP), but not other BTM, was predictive of fracture [9]. In addition, some methodological limitations should be recognized (lack of standardization of blood collection, suboptimal ascertainment of incident fractures). In a more recent study in the MrOS cohort, higher levels of urine alpha CTX isomer/Cr and greater alpha/beta CTX isomer ratio were associated with a higher risk of clinical vertebral fracture [10].

The reason for the difference between men and women is not clear. The higher bone turnover results in the deterioration of bone strength through several mechanisms: faster bone loss, increased formation of stress risers, impaired trabecular connectivity, higher fraction of bone which is only partly mineralized, and greater fraction of protein matrix which has undergone posttranslational modifications only partly. However, the "elevated" BTM levels (e.g., the highest sex-specific quartile) correspond to higher absolute BTM levels and faster bone turnover in women than men. Thus, the loss of bone strength due to faster bone turnover may be less in men than in women. In addition, in men, this smaller decline in bone strength occurs in bones which are larger and stronger. Thus, the loss of bone strength due to the faster bone turnover may have a smaller impact on the overall fracture risk in men than in women.

\section{Limitations and Potential Interest of BTM in the Clinical Practice}

Analytical and preanalytical variability have a strong effect on the BTM levels. The analytical variability depends on the BTM, the measurement method, and the technician's expertise.

The preanalytical variability comprises many factors. Circadian rhythm has a strong impact on the BTM variability, mainly serum bone resorption markers (CTX-I) [11]. Bone turnover is influenced by the vitamin $\mathrm{D}$ and calcium status, mainly in the elderly. BTM levels are increased especially in the institutionalized and home-bound vitamin D-deficient elderly who have secondary hyperparathyroidism associated with markedly increased BTM levels [12]. The seasonal variation of sunlight exposure leads to lower vitamin $\mathrm{D}$ level in winter. Consequently, levels of parathyroid hormone and BTM are higher in winter, mainly in the elderly.

Furthermore, BTM levels are influenced by a recent fracture, especially during the first 4 months [13]. GnRH agonists (used in the treatment of prostate cancer) inhibit the androgen secretion and decrease the $17 \beta$-œstradiol level leading thereby to a higher bone turnover and bone loss.

In some clinical situations, BTM levels may help to establish diagnosis. For instance, BTMs are elevated in hyperthyroidism, primary hyperparathyroidism, or Paget's disease [1]. By contrast, BTMs are decreased in hypothyroidism, hypoparathyroidism, or hypopituitarism. BTM levels are usually increased in patients with bone metastases and their levels are correlated with the spread of bone metastases [14]. A particular situation is multiple myeloma characterized by low OC concentration and markedly increased bone resorption.

Endogenous and exogenous corticosteroids inhibit bone formation [15]. The decrease in OC level is rapid and 
followed by a delayed and milder decrease in PICP and PINP. Bone resorption can increase transiently. Inhaled corticosteroids decrease OC levels in a dose- and drug-dependent manner without significant effect on other BTMs [16].

\section{Effect of Antiosteoporotic Treatment on BTM in Men}

5.1. Testosterone Replacement Therapy (TRT). In most, but not all, studies, TRT decreased bone resorption marker levels [17-21]. However, decrease in the urinary excretion of bone resorption markers per mg urinary creatinine is partly related to the increase in muscle mass, and this result should be interpreted cautiously. Bone formation markers (especially osteocalcin and $\mathrm{N}$-terminal type I collagen propeptide (P1NP)) increase slightly during the first weeks of TRT [18, 20]. This increase may reflect the direct stimulatory effect of TRT on osteoblasts in preexisting bone remodeling units. Later on, bone formation markers decrease, what reflects the general slowdown in bone turnover [20, 21]. However, this trend has not been found consistently [19].

Overall, data on the effect of TRT on BTM levels are discordant. The effect of TRT on bone turnover in hypogonadal men depends on factors such as the initial hormonal status (severity and duration of hypogonadism, hypogonadism developed in childhood or in adult age, and isolated hypogonadism or multihormonal deficit), baseline bone turnover rate, intramuscular or transdermal route of TRT administration, and normalization of testosterone level during treatment and treatment duration [22]. Testosterone increases the collagen synthesis in other tissues (e.g., muscles), which is confirmed by an increase in the serum level of N-terminal propeptide of type III collagen [23]. Thus, the potential contribution of muscle collagen to the circulating PINP and PICP levels is possible. Methodological limitations of certain studies should be recognized (small heterogeneous groups, lack of control group, large dropout, various doses of testosterone analyzed jointly, and impossibility to assess adherence to treatment).

5.2. Antiresorptive Treatment. Bisphosphonates are potent inhibitors of bone resorption. The studies in men concern mainly alendronate, risedronate, ibandronate, and zoledronate. After a given type of bisphosphonate, changes in BTM levels were similar in various groups of men with primary and secondary osteoporosis. Alendronate $(10 \mathrm{mg}$ daily per os) decreased bone resorption marker levels by about $50 \%$ after 3 months of treatment, what was followed by a milder (30 to $40 \%$ ) decrease in the serum levels of bone formation markers [24-26]. Similar trends were observed in men with primary or secondary osteoporosis related to hypogonadism or corticotherapy.

Risedronate ( 2.5 or $5 \mathrm{mg}$ daily or $35 \mathrm{mg}$ weekly per os) decreased bone resorption markers by 40 to $50 \%$ after 3 months of treatment, what was followed by a milder (25$30 \%$ ) decrease in serum levels of bone formation markers. Similar trends were observed in osteoporotic men aged 30 and over, older Japanese men with untreated osteoporosis, men on corticosteroid therapy, elderly men after stroke and elderly men with Parkinson's disease [27-31].

In 14 patients with Klinefelter's syndrome, intravenous ibandronate ( $2 \mathrm{mg}$ every 3 months) decreased urinary levels of bone resorption markers by 30 to $70 \%$ after one month [32]. This decrease was followed by a milder decrease (25$35 \%)$ in bone formation markers. In men with low aBMD (STRONG study), once-monthly oral ibandronate $(150 \mathrm{mg}$ ) induced a 40 to $50 \%$ decrease in serum concentration of Cterminal telopeptide of type I collagen (CTX-I) accompanied by a $20-30 \%$ decrease in bone alkaline phosphatase (BAP) activity [33].

BTMs were assessed in several studies carried out in men treated with zoledronate $(5 \mathrm{mg}$ i.v. once yearly in men with primary osteoporosis or hypogonadism, $4 \mathrm{mg}$ i.v. once yearly in HIV-infected men with low aBMD, single dose i.v. $4 \mathrm{mg}$ in osteopenic cancer survivors) [34-36]. Zoledronate induced a profound decrease $(>60 \%)$ in bone resorption markers within the first week of treatment followed by a milder (30$40 \%$ ) decrease in bone formation markers.

Finally, antiresorptive medications were assessed in men receiving androgen-deprivation therapy for prostate cancer. In this group, zoledronate, denosumab (monoclonal anti-RANKL antibody), and toremifene (selective estrogen receptor modulator) decreased significantly levels of bone resorption and bone formation markers [37-39].

5.3. Treatment with Bone Formation-Stimulating Agents. Recombinant human parathyroid hormone (1-34) (rhPTH$(1-34))$ induced a rapid increase in serum concentrations of bone formation markers, especially P1NP, followed by an increase in bone resorption marker levels [40, 41]. After 6 to 9 months of treatment, BTM attained the maximum (50 to $250 \%$ above baseline), then slightly decreased, but remained elevated. By contrast, during combined treatment (alendronate and rhPTH-(1-34)) started 6 months after the beginning of the anti-resorptive treatment, BTM levels were lower and the increase in $\mathrm{aBMD}$ at the spine and femoral neck was smaller than after rhPTH-(1-34) alone [41].

\section{Summary, Conclusions, and Perspectives}

Overall, in older men, higher BTM levels are associated with lower aBMD and faster bone loss. Thus, in older men, accelerated bone turnover is probably a major determinant of bone loss and osteoporosis. There are two major directions for the further studies on BTM in men. From the scientific point of view, development of markers reflecting qualitative properties of bone would improve our understanding of the mechanisms of bone fragility in older men. From the practical point of view, BTMs do not improve the identification of men at high risk of accelerated bone loss or fragility fracture. In older men, changes in BTM levels induced by antiosteoporotic treatment are similar to those found in postmenopausal women. However, we need more studies on the association between changes in bone turnover rate and the decrease in the risk of fragility fracture. In particular, we need studies on the utility of BTM measurement for the improvement of the persistence and adherence to the anti-osteoporotic treatment in men. 


\section{References}

[1] P. Szulc, J. M. Kaufman, and P. D. Delmas, "Biochemical assessment of bone turnover and bone fragility in men," Osteoporosis International, vol. 18, no. 11, pp. 1451-1461, 2007.

[2] S. Vasikaran, C. Cooper, R. Eastell et al., "International Osteoporosis Foundation and International Federation of Clinical Chemistry and Laboratory Medicine Position on bone marker standards in osteoporosis," Clinical Chemistry and Laboratory Medicine, vol. 49, no. 8, pp. 1271-1274, 2011.

[3] P. Szulc, P. Garnero, F. Munoz, F. Marchand, and P. D. Delmas, "Cross-sectional evaluation of bone metabolism in men," Journal of Bone and Mineral Research, vol. 16, no. 9, pp. 16421650, 2001.

[4] D. L. Schneider and E. L. Barrett-Connor, "Urinary N-telopeptide levels discriminate normal, osteopenic, and osteoporotic bone mineral density," Archives of Internal Medicine, vol. 157, no. 11, pp. 1241-1245, 1997.

[5] P. Szulc, A. Montella, and P. D. Delmas, "High bone turnover is associated with accelerated bone loss but not with increased fracture risk in men aged 50 and over: the prospective MINOS study," Annals of the Rheumatic Diseases, vol. 67, no. 9, pp. 1249-1255, 2008.

[6] D. C. Bauer, P. Garnero, S. L. Harrison et al., "Biochemical markers of bone turnover, hip bone loss, and fracture in older men: the MrOS study," Journal of Bone and Mineral Research, vol. 24, no. 12, pp. 2032-2038, 2009.

[7] E. Dennison, R. Eastell, C. H. D. Fall, S. Kellingray, P. J. Wood, and C. Cooper, "Determinants of bone loss in elderly men and women: a prospective population-based study," Osteoporosis International, vol. 10, no. 5, pp. 384-391, 1999.

[8] H. Luukinen, S. M. Kakonen, K. Pettersson et al., "Strong prediction of fractures among older adults by the ratio of carboxylated to total serum osteocalcin," Journal of Bone and Mineral Research, vol. 15, no. 12, pp. 2473-2478, 2000.

[9] C. Meier, T. V. Nguyen, J. R. Center, M. J. Seibel, and J. A. Eisman, "Bone resorption and osteoporotic fractures in elderly men: the Dubbo Osteoporosis Epidemiology study," Journal of Bone and Mineral Research, vol. 20, no. 4, pp. 579587, 2005.

[10] D. Bauer, P. Garnero, S. Litwack Harrison et al., “Type I collagen isomerization (Alpha/Beta CTX Ratio) and risk of clinical vertebral fracture in men: a prospective study," http://www .asbmr.org/Meetings/AnnualMeeting/AbstractDetail.aspx? aid=7ed933e3-0487-4b5a-b2dd-747876d1ecde.

[11] P. Qvist, S. Christgau, B. J. Pedersen, A. Schlemmer, and C. Christiansen, "Circadian variation in the serum concentration of C-terminal telopeptide of type I collagen (serum CTx): effects of gender, age, menopausal status, posture, daylight, serum cortisol, and fasting," Bone, vol. 31, no. 1, pp. 57-61, 2002.

[12] R. Theiler, H. B. Stähelin, M. Kränzlin, A. Tyndall, and H. A. Bischoff, "High bone turnover in the elderly," Archives of Physical Medicine and Rehabilitation, vol. 80, no. 5, pp. 485-489, 1999.

[13] K. Stoffel, H. Engler, M. Kuster, and W. Riesen, "Changes in biochemical markers after lower limb fractures," Clinical Chemistry, vol. 53, no. 1, pp. 131-134, 2007.

[14] D. J. Leeming, M. Koizumi, I. Byrjalsen, B. Li, P. Qvist, and L. B. Tankó, "The relative use of eight collagenous and noncollagenous markers for diagnosis of skeletal metastases in breast, prostate, or lung cancer patients," Cancer Epidemiology Biomarkers and Prevention, vol. 15, no. 1, pp. 32-38, 2006.
[15] A. Dovio, L. Perazzolo, G. Osella et al., "Immediate fall of bone formation and transient increase of bone resorption in the course of high-dose, short-term glucocorticoid therapy in young patients with multiple sclerosis," Journal of Clinical Endocrinology and Metabolism, vol. 89, no. 10, pp. 4923-4928, 2004.

[16] F. Richy, J. Bousquet, G. E. Ehrlich et al., "Inhaled corticosteroids effects on bone in asthmatic and COPD patients: a quantitative systematic review," Osteoporosis International, vol. 14, no. 3, pp. 179-190, 2003.

[17] A. M. Kenny, A. Kleppinger, K. Annis et al., "Effects of transdermal testosterone on bone and muscle in older men with low bioavailable testosterone levels, low bone mass, and physical frailty," Journal of the American Geriatrics Society, vol. 58, no. 6, pp. 1134-1143, 2010.

[18] C. Y. Guo, T. H. Jones, and R. Eastell, "Treatment of isolated hypogonadotropic hypogonadism effect on bone mineral density and bone turnover," Journal of Clinical Endocrinology and Metabolism, vol. 82, no. 2, pp. 658-665, 1997.

[19] J. K. Amory, N. B. Watts, K. A. Easley et al., "Exogenous testosterone or testosterone with finasteride increases bone mineral density in older men with low serum testosterone," Journal of Clinical Endocrinology and Metabolism, vol. 89, no. 2, pp. 503-510, 2004.

[20] C. Wang, R. S. Swerdloff, A. Iranmanesh et al., "Effects of transdermal testosterone gel on bone turnover markers and bone mineral density in hypogonadal men," Clinical Endocrinology, vol. 54, no. 6, pp. 739-750, 2001.

[21] P. J. Snyder, H. Peachey, J. A. Berlin et al., "Effects of testosterone replacement in hypogonadal men," Journal of Clinical Endocrinology and Metabolism, vol. 85, no. 8, pp. 2670-2677, 2000.

[22] A. M. Isidori, E. Giannetta, E. A. Greco et al., "Effects of testosterone on body composition, bone metabolism and serum lipid profile in middle-aged men: a meta-analysis," Clinical Endocrinology, vol. 63, no. 3, pp. 280-293, 2005.

[23] S. Bhasin, E. J. He, M. Kawakubo et al., "N-terminal propeptide of type III procollagen as a biomarker of anabolic response to recombinant human GH and testosterone," Journal of Clinical Endocrinology and Metabolism, vol. 94, no. 11, pp. 4224-4233, 2009.

[24] E. Orwoll, M. Ettinger, S. Weiss et al., "Alendronate for the treatment of osteoporosis in men," New England Journal of Medicine, vol. 343, no. 9, pp. 604-610, 2000.

[25] K. G. Saag, R. Emkey, T. J. Schnitzer et al., "Alendronate for the prevention and treatment of glucocorticoid-induced osteoporosis," New England Journal of Medicine, vol. 339, no. 5, pp. 292-299, 1998.

[26] I. Shimon, V. Eshed, R. Doolman, B. A. Sela, A. Karasik, and I. Vered, "Alendronate for osteoporosis in men with androgenrepleted hypogonadism," Osteoporosis International, vol. 16, no. 12, pp. 1591-1596, 2005.

[27] S. Boonen, E. S. Orwoll, D. Wenderoth, K. J. Stoner, R. Eusebio, and P. D. Delmas, "Once-weekly risedronate in men with osteoporosis: results of a 2-Year, placebo-controlled, double-blind, multicenter study," Journal of Bone and Mineral Research, vol. 24, no. 4, pp. 719-725, 2009.

[28] T. Majima, A. Shimatsu, Y. Komatsu et al., "Effects of risedronate or alfacalcidol on bone mineral density, bone turnover, back pain, and fractures in Japanese men with primary osteoporosis: results of a two-year strict observational study," Journal of Bone and Mineral Metabolism, vol. 27, no. 2, pp. 168-174, 2009. 
[29] D. M. Reid, S. Adami, J. P. Devogelaer, and A. A. Chines, "Risedronate increases bone density and reduces vertebral fracture risk within one year in men on corticosteroid therapy," Calcified Tissue International, vol. 69, no. 4, pp. 242247, 2001.

[30] Y. Sato, J. Iwamoto, T. Kanoko, and K. Satoh, "Risedronate sodium therapy for prevention of hip fracture in men 65 years or older after stroke," Archives of Internal Medicine, vol. 165, no. 15, pp. 1743-1748, 2005.

[31] Y. Sato, Y. Honda, and J. Iwamoto, "Risedronate and ergocalciferol prevent hip fracture in elderly men with Parkinson disease," Neurology, vol. 68, no. 12, pp. 911-915, 2007.

[32] J. J. Stepan, P. Burckhardt, and V. Hána, “The effects of threemonth intravenous ibandronate on bone mineral density and bone remodeling in Klinefelter's syndrome: the influence of vitamin D deficiency and hormonal status," Bone, vol. 33, no. 4, pp. 589-596, 2003.

[33] E. S. Orwoll, N. C. Binkley, E. M. Lewiecki, U. Gruntmanis, M. A. Fries, and G. Dasic, "Efficacy and safety of monthly ibandronate in men with low bone density," Bone, vol. 46, no. 4, pp. 970-976, 2010.

[34] E. S. Orwoll, P. D. Miller, J. D. Adachi et al., "Efficacy and safety of a once-yearly i.v. infusion of zoledronic acid $5 \mathrm{mg}$ versus a once-weekly 70-mg oral alendronate in the treatment of male osteoporosis: a randomized, multicenter, double-blind, activecontrolled study," Journal of Bone and Mineral Research, vol. 25, no. 10, pp. 2239-2250, 2010.

[35] M. J. Bolland, A. B. Grey, A. M. Horne et al., "Annual zoledronate increases bone density in highly active antiretroviral therapy-treated human immunodeficiency virus-infected men: a randomized controlled trial," Journal of Clinical Endocrinology and Metabolism, vol. 92, no. 4, pp. 1283-1288, 2007.

[36] J. E. Brown, S. P. Ellis, J. E. Lester et al., "Prolonged efficacy of a single dose of the bisphosphonate zoledronic acid," Clinical Cancer Research, vol. 13, no. 18, pp. 5406-5410, 2007.

[37] C. W. Ryan, D. Huo, L. M. Demers, T. M. Beer, and L. V. Lacerna, "Zoledronic acid initiated during the first year of androgen deprivation therapy increases bone mineral density in patients with prostate cancer," Journal of Urology, vol. 176, no. 3, pp. 972-978, 2006.

[38] M. R. Smith, B. Egerdie, N. H. Toriz et al., "Denosumab in men receiving androgen-deprivation therapy for prostate cancer," New England Journal of Medicine, vol. 361, no. 8, pp. 745-755, 2009.

[39] M. R. Smith, R. A. Morton, K. G. Barnette et al., “Toremifene to reduce fracture risk in men receiving androgen deprivation therapy for prostate cancer," Journal of Urology, vol. 184, no. 4, pp. 1316-1321, 2010.

[40] E. S. Orwoll, W. H. Scheele, S. Paul et al., "The effect of teriparatide [human parathyroid hormone (1-34)] therapy on bone density in men with osteoporosis," Journal of Bone and Mineral Research, vol. 18, no. 1, pp. 9-17, 2003.

[41] E. S. Kurland, F. Cosman, D. J. McMahon, C. J. Rosen, R. Lindsay, and J. P. Bilezikian, "Parathyroid hormone as a therapy for idiopathic osteoporosis in men: effects on bone mineral density and bone markers," Journal of Clinical Endocrinology and Metabolism, vol. 85, no. 9, pp. 3069-3076, 2000. 


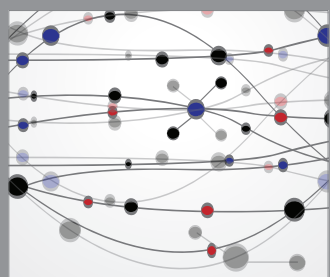

The Scientific World Journal
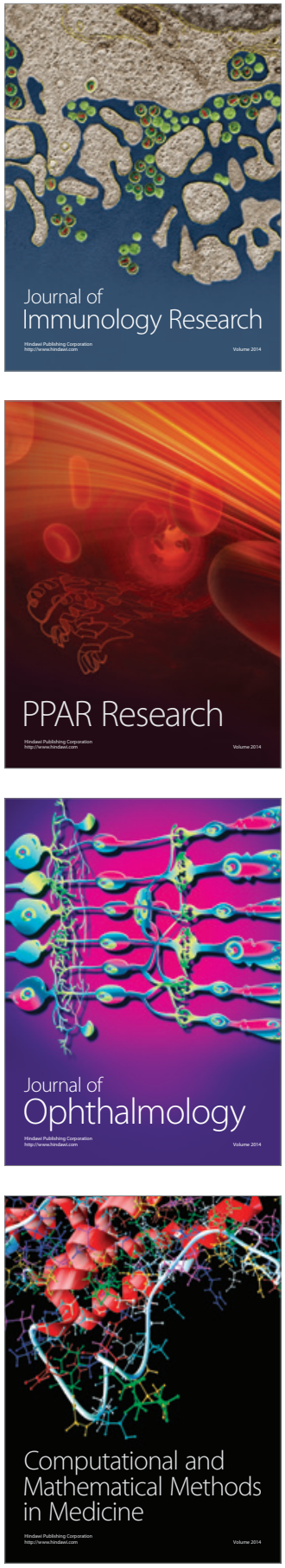

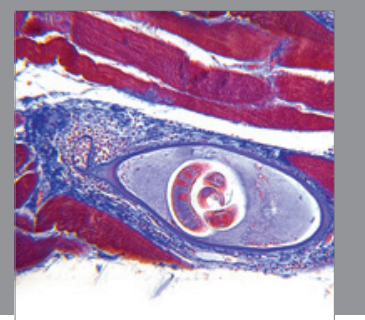

Gastroenterology

Research and Practice
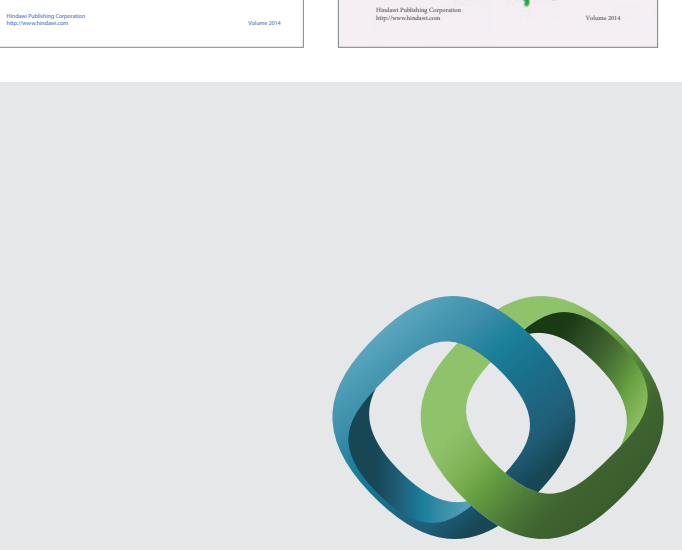

\section{Hindawi}

Submit your manuscripts at

http://www.hindawi.com
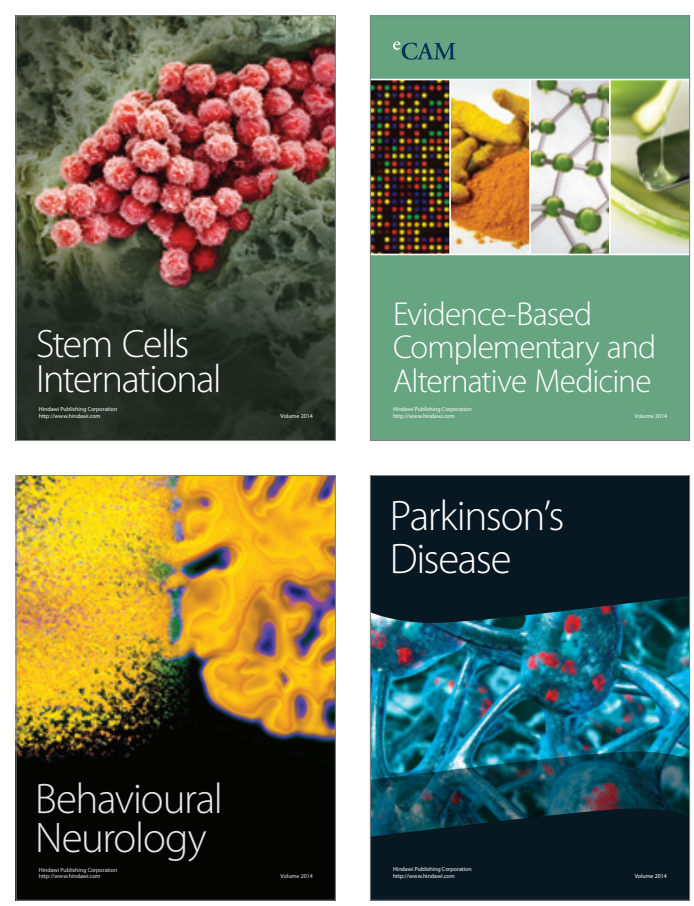

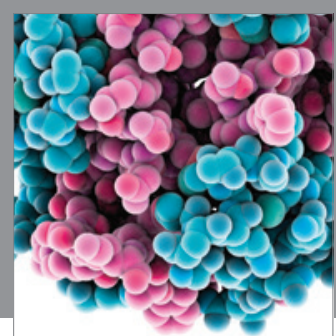

Journal of
Diabetes Research

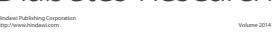

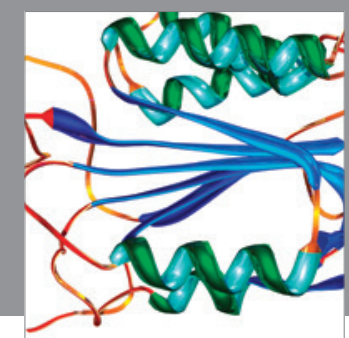

Disease Markers
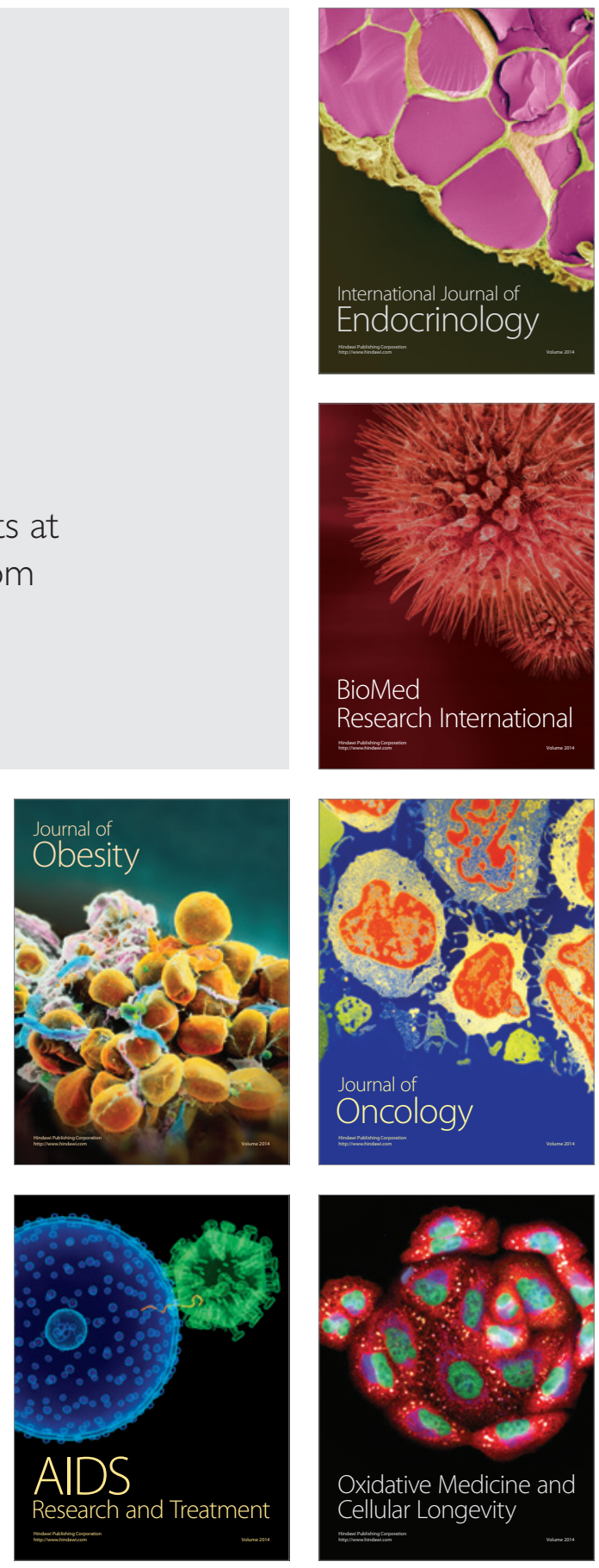